\title{
Spectrum of Haematological malignancies managed at tertiary centre: A one-year retrospective review
}

\author{
Anjan Shrestha ${ }^{1}$, Shovana Thapa ${ }^{1}$, Usha Mnandhar ${ }^{1}$, Gita Sayami ${ }^{1}$, Pradeep K Shrestha ${ }^{2}$, Yogendra P Singh ${ }^{3}$ \\ ${ }^{1}$ Department of Pathology, Institute of Medicine, Tribhuwan University Teaching Hospital, Nepal \\ ${ }^{2}$ Department of Medicine, Institute of Medicine, Tribhuwan University Teaching Hospital, Nepal \\ ${ }^{3}$ Department of Surgery, Institute of Medicine, Tribhuwan University Teaching Hospital, Nepal \\ Correspondence: Dr Anjan Shrestha, Department of Pathology, Institute of Medicine, Tribhuwan University Teaching Hospital, Nepal.
}

\begin{abstract}
Introduction: Hematological malignancies are not uncommon and all ages and genders are affected. Hematological malignancies are a group of cancers that arise from a malignant transformation of cells of the bone marrow or lymphatic system. There are several classification systems for Hematological Malignancies. The WHO classification was the first worldwide consensus classification on hematological tumors.

Methods: We retrospectively collected data from the Department of Pathology and Hematology Unit of Internal medicine from 2015 to 2016 . Hematological malignancies were analyzed clinically and with laboratory parameters. They were initially analyzed with complete blood counts and peripheral smear and diagnosed on the basis of Bone marrow morphological assessment, Immunophenotyping and cytogenetic and molecular markers and histopathology and Immunohistochemistry of Excised lymph node when applicable. Plasma cell dyscrasias were assessed clinically for features of CRAB (hypercalcemia, renal impairment, anemia and lytic lesion) and evaluation was done by hemogram, biochemical parameters and skeletal survey. Later plasma cell dyscrasias was diagnosed on the basis of bone marrow study, Immunofixation electrophoresis, serum free light chain assay, serum protein electrophoresis and myeloma defining events.
\end{abstract}

Results: There were 110 cases of hematological neoplasm from Feb 2015 till Jan 2016. Lymphoid neoplasm was the commonest hematological malignancies with $60.9 \%$ followed by myeloid neoplasm of $37.3 \%$ then histiocytic neoplasm of $1.5 \%$. Median age at diagnosis for all Hematological malignancies was 55 years of age. In patients under 20 years of age, T ALL and LCH accounted each with $0.9 \%$. In young adult Patient, NHL was the most common HM whereas; MPN was the most common HM in adults. In older patients, PCN was the most common HM.

Male were more prevalent than female in Hematological malignancies. Male accounted for $73.6 \%$ and female were $26.4 \%$ with male female ratio $2.7: 1$. In both male and female, Lymphoid Neoplasm was most frequent HM. In male PCN was the most frequent HM. In female, NHL, PCN and MPN was the most frequent HM.

Lymphoid neoplasm included Mature B cell Neoplasm (MBCN) 43.6\%, Acute Lymphoblastic Leukemia (ALL) 7.3\%, Hodgkins Lymphoma (HL) 5.5\% and Mature T cell Neoplasm (MTCN) $3.6 \%$. Plasma cell Neoplasm (PCN) (29\%) was the commonest lymphoid neoplasm. Among the myeloid neoplasm (MN), Myeloproliferative neoplasm (MPN) accounted $19.1 \%$ followed by acute myeloid leukemia (AML) with 14.5\% then Myelodysplastic syndrome (MDS) 4.5\%. Chronic Myeloid Leukemia (CML) is the commonest HM among different subtypes of MPN.

Conclusion: HM can occur at any age group with median age at diagnosis of 55 years. Overall men are more affected with HM than women. In both male and female Lymphoid malignancies are frequent HM. There is difference in distribution pattern and subtypes of Hematological malignancies at different age group.

Keywords: Haematological malignancies; Lymphoid neoplasm; Myeloid neoplasm. 


\section{Introduction}

Hematological malignancies (HM) are not uncommon in our country. All ages and genders are affected. ${ }^{1}$ Hematological Malignancies are a group of cancers that arise from a malignant transformation of cells of the bone marrow or lymphatic system. ${ }^{2}$ There are several classification systems for Hematological Malignancies. The 2001 WHO classification was the first worldwide consensus classification on hematological tumors. The classification is based on information such as clinical, morphologic, biologic, immunophenotypic and genetic features. $3,4,5,6$ In 2008, as part of series of Classification of Tumours 'blue book' monographs ( $4^{\text {th }}$ edition), the WHO published a new classification for hematopoetic and lymphoid neoplasm in collaboration with society for Hematopathology and the European Association for Haematopathology. In 2014, a clinical advisory committee (CAC) proposed revisions to fourth edition of classification. So, In view of recently identified molecular features, improvisation of morphological features and integrated approach, fourth edition is being updated in 2016. ${ }^{5,6}$ Lymphoid Neoplasm are classified as Mature B cell Neoplasm (MBCN), mature T and NK cell Neoplasm (MTCN), Post transplant Lymphoproliferative Disorders, Hodgkins Disease (HL) and Histiocytic and dendritic Cell Neoplasm (HDN). Myeloid Neoplasm are classified as Myeloproliferative Neoplasms (MPN), Myeloid/Lymphoid Neoplasms with eosinophilia with rearrangement, Myelodysplastic/Myeloproliferative Neoplasms, Myelodysplastic Syndrome (MDS), Acute myeloid Leukemia with related neoplasms(AML), blastic plasmacytoid dendritic cell Neoplasms, Acute Leukemia with ambigious lineage, B and T lymphoblastic Lymphoma/ Leukemia (ALL). ${ }^{5,6}$

In Institute of Medicine, Maharajgunj Medical Campus, Kathmandu, Nepal, there is no database on hematological cancers. After initiation of Haemato-oncology unit in the year 2015 analysis of Hematological malignancies in the Institute was conducted.

\section{Methods}

We retrospectively collected data from the Department of Pathology and Hematology Unit of Internal medicine from 2015 to 2016. Hematological malignancies were analyzed clinically and with laboratory parameters. Patients diagnosed as Leukemia, Myeloproliferative Neoplasm and Myelodysplastic Syndrome were initially analyzed with complete blood counts and peripheral smear and diagnosed on the basis of Bone marrow morphological assessment, Immunophenotyping and cytogenetic and molecular markers in some cases. After clinical examination Lymphomas were diagnosed on the basis of histopathology and Immunohistochemistry of Excised lymph node. Plasma cell dyscrasias were assessed clinically for features of CRAB (hypercalcemia, renal impairment, anemia and lytic lesion) and evaluation was done by hemogram, biochemical parameters and skeletal survey. Later plasma cell dyscrasias was diagnosed on the basis of bone marrow study, Immunofixation electrophoresis, serum free light chain assay, serum protein electrophoresis and myeloma defining events. Ancillary techniques (immunophenotyping, cytogenetics, immunofixation electrophoresis, serum free light chain assay, molecular markers) that were not available in our Institute were outsourced for diagnosis. Clinical information of patients was received from admission file of department of medicine and different laboratory parameters were retrieved from stored data from department of pathology.

\section{Results}

In this study, we had 110 cases of hematological neoplasm (HN) from Feb 2015 till Feb 2016. Lymphoid neoplasm was the commonest hematological malignancies with 67 cases $(60.9 \%)$ followed by myeloid neoplasm of 41 cases $(37.3 \%)$ and Histiocytic neoplasm of 2 cases $(1.5 \%)$. Overall median age at diagnosis for all Hematological malignancies was 55 years of age. We have excluded patient of less than 16 years of age.

Lymphoid neoplasm included MBCN 43.6\%, ALL $7.3 \%$, HL 5.5\% and MTCN 3.6\%. In this study, Plasma cell Neoplasm (PCN) (29\%) was the commonest lymphoid neoplasm followed respectively by Non Hodgkin Lymphoma (NHL) 14.5\%, ALL 7.3\%, HL 5.5\%, Chronic Lymphocytic Leukemia (CLL) 2.7\% and Lymphoplasmacytic Lymphoma (LPL) $0.9 \%$. NHL includes Diffuse Large B cell Lymphoma (DLBCL)(5.5\%), Follicular Lymphoma (FL)(1.8\%), Mantle cell Lymphoma (MCL) $(0.9 \%)$, Marzinal Zone Lymphoma (MZL)(0.9\%), Primary Effusion Lymphoma (PEL) $(0.9 \%)$, Primary Mediastinal B cell Lymphoma (PMBCL) $(0.9 \%)$, Primary Cutaneous Anaplastic Large Cell Lymphoma (PCALCL) (0.9\%), Hepatosplenic T cell Lymphoma (HSTCL) (0.9\%), Peripheral T cell Lymphoma (PTCL) (0.9\%), Adult T cell Lymphoma (ATCL) (0.9\%). (Table 1) 
Table 1 Frequency and Distribution pattern of age (years) and sex in Lymphoid Neoplasm

\begin{tabular}{|c|c|c|c|c|c|c|c|c|}
\hline $\mathbf{H} \mathbf{M}$ & $110(100 \%)$ & $10-20$ & $21-40$ & $41-60$ & $>60$ years & male & female & M:F \\
\hline A.Lymphoid Neoplasm & $66(60 \%)$ & $1(0.9 \%)$ & $16(14.5$ & $31(28 . \%$ & $18(16.3 \%$ & $50(45.4 \%$ & $16(14.5$ & $3.1: 1$ \\
\hline 1.Precuorsor Lymphoid & d $8(7.27 \%)$ & $1(0.9 \%)$ & $4(3.6 \%)$ & $3(2.7 \%)$ & - & $7(6.36 \%)$ & $1(0.9 \%)$ & $7: 1$ \\
\hline a. B-ALL & $4(3.6 \%)$ & - & $3(2.7 \%)$ & $1(0.9 \%)$ & - & $4(3.6 \%)$ & - & - \\
\hline b. T-ALL & $4(3.6 \%)$ & $1(0.9 \%)$ & $1(0.9 \%)$ & $27(1.8 \%)$ & - & $3(2.7 \%)$ & $1(0.9 \%)$ & $3: 1$ \\
\hline 2.MBCN & $48(43.6 \% \%)$ & - & $3(2.7 \%)$ & $5(4.5 \%)$ & $18(16.3 \%$ & $36(32.7 \%$ & $12(10.9$ & $3: 1$ \\
\hline a. NHL & $12(10.9 \%)$ & - & $3(2.7 \%)$ & $2(1.8 \%)$ & $4(3.6 \%)$ & $8(7.27 \%)$ & $4(3.6 \%)$ & $2: 1$ \\
\hline a1. FL & $2(1.8 \%)$ & - & - & - & - & $1(0.9 \%)$ & $1(0.9 \%)$ & $1: 1$ \\
\hline a2. MZL & $1(0.9 \%)$ & - & - & $3(2.7 \%)$ & $1(0.9 \%)$ & $1(0.9 \%)$ & - & - \\
\hline a3. DLBCL & $6(5.5 \%)$ & - & $2(1.8 \%)$ & - & $1(0.9 \%)$ & $4(3.6 \%)$ & $2(1.8 \%)$ & $2: 1$ \\
\hline a4. PMBCL & $1(0.9 \%)$ & - & $1(0.9 \%)$ & - & - & - & $1(0.9 \%)$ & - \\
\hline a5. PEL & $1(0.9 \%)$ & - & - & - & $1(0.9 \%)$ & $1(0.9 \%)$ & - & - \\
\hline \multirow[t]{2}{*}{ a6. MCL } & $1(0.9 \%)$ & - & - & & $1(0.9 \%)$ & $1(0.9 \%)$ & - & - \\
\hline & & & & $1(0.9 \%)$ & & & & \\
\hline b. LPL & $1(0.9 \%)$ & - & - & $1(0.9 \%)$ & - & - & $1(0.9 \%)$ & - \\
\hline c. CLL & $3(2.7 \%)$ & - & - & $20(18 \%)$ & $2(1.8 \%)$ & $2(1.8 \%)$ & $1(0.9 \%)$ & $2: 1$ \\
\hline d. PCN & $32(29 \%)$ & - & - & & $12(10.9 \%$ & $26(23.6 \%$ & $6(5.5 \%)$ & 4.3:1 \\
\hline 3.MTCN (NHL) & $4(3.6 \%)$ & & & & & & & \\
\hline a. PCALCL & $1(0.9 \%)$ & - & $4(3.6 \%)$ & - & - & $3(2.7 \%)$ & $1(0.9 \%)$ & $3: 1$ \\
\hline b. HSTCL & $1(0.9 \%)$ & - & $1(0.9 \%)$ & - & - & $1(0.9 \%)$ & - & - \\
\hline c. PTCL, NOS & $1(0.9 \%)$ & - & $1(0.9 \%)$ & - & - & $1(0.9 \%)$ & - & - \\
\hline \multirow[t]{2}{*}{ d.ATCL } & $1(0.9 \%)$ & - & $1(0.9 \%)$ & - & - & - & $1(0.9 \%)$ & - \\
\hline & & - & $1(0.9 \%)$ & - & & $1(0.9 \%)$ & - & - \\
\hline 4. HL & $6(5.5 \%)$ & - & $5(4.5 \%)$ & $1(0.9 \%)$ & - & $4(3.6 \%)$ & $2(1.8 \%)$ & $2: 1$ \\
\hline
\end{tabular}

Among the Myeloid neoplasm (MN), Myeloproliferative neoplasm (MPN) accounted for the maximum number of 9.1\% followed by acute myeloid leukemia and precursor neoplasm (AML) of 14.5\% then Myelodysplastic syndromes (MDS) 4.5\%. AML with recurrent genetic abnormalities were seen in 3.6\% where as AML NOS were 10.9\%. In this study, out of 21 MPN cases, Chronic Myeloid Leukemia (CML) were maximum number of cases accounting for 11.8\% cases followed by Essential Thrombocythaemia(ET) $3.6 \%$ and Polycythemia Vera (PV)1.8\% and Primary Myelofibrosis (PM)1.8\%. Among MDS, Refractory cytopenia with unilineage Dysplasia (RCUD) and Refractory Cytopenia with multilineage Dysplasia (RCMD) were equal number of cases that is $1.8 \%$ where as Refractory Anemia with Excess Blast (RAEB) was 0.9\%. (Table 2) 
Table 2 Frequency and Distribution pattern of age (years) and sex in Myeloid Neoplasm

\begin{tabular}{|c|c|c|c|c|c|c|c|}
\hline HM & $110(100 \%)$ & $21-40$ yrs & $41-60$ yrs & $>61 \mathrm{yrs}$ & Male & female & M:F \\
\hline A.Myeloid Neoplasm & $42(38.1 \%)$ & $10(9.09 \%)$ & $25(22.7 \%)$ & $7(6.36 \%)$ & $29(26.3 \%)$ & $13(11.8 \%)$ & $2.2: 1$ \\
\hline 1. AML & $16(14.5 \%)$ & $5(4.5 \%)$ & $7(6.36 \%)$ & $4(3.36 \%)$ & $12(10.9 \%)$ & $4(3.6 \%)$ & $3: 1$ \\
\hline 2.MPN & $21(19.1 \%)$ & $5(4.5 \%)$ & $14(12.7 \%)$ & $2(1.8 \%)$ & $15(13.6 \%)$ & $6(5.5 \%)$ & $2.5: 1$ \\
\hline a. CML & $13(11.8 \%)$ & $5(4.5 \%)$ & $7(6.3 \%)$ & $1(0.9 \%)$ & $8(7.27 \%)$ & $5(4.5 \%)$ & $1.6: 1$ \\
\hline b. PV & $2(1.8 \%)$ & - & $2(1.8 \%)$ & - & $2(1.8 \%)$ & - & - \\
\hline c. ET & $4(3.6 \%)$ & - & $3(2.7 \%)$ & $1(0.9 \%)$ & $3(2.7 \%)$ & $1(0.9 \%)$ & $3: 1$ \\
\hline d. PM & $2(1.8 \%)$ & - & $2(1.8 \%)$ & - & $2(1.8 \%)$ & - & - \\
\hline 3. MDS & $5(4.5 \%)$ & - & $4(3.6 \%)$ & $1(0.9 \%)$ & $2(1.8 \%)$ & $3(2.7 \%)$ & \multirow{2}{*}{$1: 1.5$} \\
\hline a. RCUD & $2(1.8 \%)$ & - & $2(1.8 \%)$ & - & $2(1.8 \%)$ & - & \\
\hline b. RCMD & $2(1.8 \%)$ & - & $1(0.9 \%)$ & $1(0.9 \%)$ & - & $2(1.8 \%)$ & \\
\hline c. RAEB & $1(0.9 \%)$ & - & $1(0.9 \%)$ & - & - & $1(0.9 \%)$ & \\
\hline
\end{tabular}

Histiocytic and dendritic cell neoplasm (HDN) accounted for 2 cases $(1.5 \%)$. Between 2 cases $(1.5 \%)$ of histiocytic and dendritic Neoplasm were Langerhans cell histiocytosis.

We had two Patients less than 20 years of age, T ALL and LCH was seen accounting each $0.9 \%$ of HM. In young adult Patient of 21 to 40 years of age, NHL was the most common HM with 9.09\% followed by AML 4.5\%, MPN (CML) 4.5\%, B ALL $2.7 \%$, T ALL $0.9 \%$, HL $0.9 \%$ and LCH $0.9 \%$.

In adults aged 41 to 60 years, MPN 12.6\% (CML 6.3\%, ET $2.7 \%$, PV1.8\%, PM1.8\%) was the most common HM followed respectively by PCN accounting $10.9 \%$ followed by AML 7.2\%, NHL 4.5\%, MDS 3.6\%, ALL 1.8\%, HL $0.9 \%$, CLL $1(0.9 \%)$ and LPL $1(0.9 \%)$.

In older patients of more than 61 years of age, PCN was the most common HM accounting $10.9 \%$ followed by NHL $3.6 \%$, AML 2.9\%, CLL 1.8\%, MPN 1.8\%(CML 0.9\%, ET $0.9 \%$ ) and $\operatorname{MDS} 1(0.9 \%)$.

Hematological malignancies were prevalent more in male than in female. Male accounted for $73.6 \%$ and female were $26.4 \%$ with male female ratio $2.7: 1$. In both sex, Lymphoid Neoplasm was most frequent HM followed by Myeloid Neoplasm and Histiocytic Neoplasm.

In males, Lymphoid Neoplasm was most frequent HM with $41.4 \%$ followed by Myeloid Neoplasm of $26.3 \%$ and Histiocytic Neoplasm accounting 1.8\%. Among all these HM, PCN was the most frequent HM with $23.6 \%$ followed by MPN $13.57 \%$ (CML $7.27 \%$, ET $2.7 \%$, PV $1.8 \%$, PM $1.8 \%$ ), AML $0.9 \%$, NHL $10 \%$ including both B and $\mathrm{T}$ phenotype, HL 3.6\%, Myelofibrosis 1.8\%, MDS 1.8\% and CLL $1.8 \%$. However duration of study done was only 1 year and number of cases studied may be low. So, LPL was not seen in male in this study.

In female, Lymphoid Neoplasm was most frequent HM with 16 cases $(14.5 \%)$ followed by Myeloid Neoplasm of $11.8 \%$. Histiocytic Neoplasm was not seen in female in the study. Among all these Hematological malignancies, NHL, PCN and MPN was the most frequent HM with each 5.5\% followed by AML 3.6\%, MDS 2.7\%, HL 1.8\%, CLL 1.8\%, LPL $0.9 \%$ and ALL $0.9 \%$. However, PV and Myelofibrosis was not seen in female in this study.

\section{Discussion}

This is the first study on Hematological malignancies done in TUTH. It has shown the relative frequencies and distribution of several subtypes of hematological malignancies in different age group and sex. Hematological malignancies were 110 cases in the year February 2015 to January 2016. 
In this study overall median age at diagnosis for all Hematological malignancies was 55 years of age. Similarly study done in Eastern morocco also shows median age of 54 years of age. ${ }^{7}$ Study done in Bangladesh showed median age of 42 years. ${ }^{8}$ It shows there is similar age group involvement in our institute and Morocco. However, in Bangladesh median age is younger.

We have found that overall men are more affected than women, with male to female ratio of 2.7:1. The study done in Eastern Morocco, Bangladesh and Pakistan showed slight male predominance in Hematological malignancies. ${ }^{7,8,16}$ It has been known that most myeloid and lymphoid are more common in males than females with a justification for this being that men are more likely to be exposed to potentially carcinogenic occupational and environmental agents. ${ }^{15}$ In both sex Lymphoid Neoplasm was most frequent HM followed by Myeloid Neoplasm and Histiocytic Neoplasm respectively.

Here, Lymphoid neoplasm was the commonest hematological malignancy with $60.9 \%$ followed by myeloid neoplasm $37.3 \%$ and Histiocytic neoplasm (LCH) $1.5 \%$. The study done in North America, Australia, Europe and Africa, Eastern morocco and Pakistan also revealed lymphoid Neoplasm is frequent hematological malignancy. ${ }^{9-13,16}$

Among the Myeloid neoplasms, Myeloproliferative neoplasm accounted for the maximum number of cases with $19.1 \%$ followed by AML $14.5 \%$ and MDS $4.5 \%$. The study done in Eastern morocco had similar result of MPN as the commonest Myeloid Neoplasm followed by AML and MDS. ${ }^{7}$ The study done in Uttarakhand, India found AML was commonest Leukemia followed by CML. ${ }^{14}$ In our study, Lymphoid neoplasm included MBCN 43.6\%, ALL 7.3\%, HL 5.5\% and MTCN 3.6\%. In this study, PCN (29\%) was the commonest lymphoid neoplasm followed respectively by NHL $14.5 \%$, ALL $7.3 \%$, HL $5.5 \%$, CLL $2.7 \%$ and LPL $0.9 \%$. Among different subtypes of NHL diagnosed common was DLBCL 5.5\%. Study done in Eastern morocco showed NHL as the commonest lymphoid malignancy followed by HL. In that study DLBCL was the commonest NHL. ${ }^{7}$ In Pakistan and US, NHL is the commonest HM. ${ }^{8}$

In males, Lymphoid Neoplasm was most frequent $\mathrm{HM}$ with $41.4 \%$ followed by Myeloid Neoplasm of 26.3\% and Histiocytic Neoplasm accounting $1.8 \%$. PCN was the most frequent $\mathrm{HM}$ with $23.6 \%$ followed by MPN $13.57 \%$ (CML 7.27\%, ET 2.7\%, PV 1.8\%, PM 1.8\%), AML 10.9\%, NHL $10 \%$ (both B and T phenotype), HL 3.6\%, MDS $1.8 \%$ and CLL $1.8 \%$. However, a review of hematological malignancies in eastern Morocco revealed slight variation in frequency of different HM where NHL was the most frequent HM followed respectively by HL, PCN, MPN, CLL, AML, ALL, and finally MDS. ${ }^{7}$ PCN (Multiple myeloma) has been seen to affect males for frequently. ${ }^{14}$ In female also, Lymphoid Neoplasm was most frequent HM with 14.5\% followed by Myeloid Neoplasm of $11.8 \%$. Here, NHL (Including T and B phenotype), PCN and MPN (CML $4.5 \%$ and ET $0.9 \%$ ) was seen in equal frequencies with $5.5 \%$ followed by AML 3.6\%, MDS 2.7\%, HL 1.8\%, CLL $1.8 \%$, LPL $0.9 \%$ and ALL $0.9 \%$. NHL has been observed to be the most frequent HM followed by HL, PMN, LPL, AML, MDS, CLL and ALL. ${ }^{7}$ In female there is slight similar results seen in these studies however there is different frequencies of HM in Male.

In our study, patient under 20 years of age, T- ALL and LCH was seen each accounting for $0.9 \%$ of HM. However, HL has been seen to be the most common HM followed by NHL, AML, MPN and ALL. ${ }^{7}$ We had excluded cases under 16 years of age so we had less numbers of cases under 20 years of age in our study. It has been seen that Precursors Tand B-cell malignancies are primarily diseases of children and young adults. ${ }^{15}$

In young adult Patient of 21 to 40 years of age, NHL was the most common HM with $9.09 \%$ followed by AML $4.5 \%$, MPN (CML) 4.5\%, B ALL 2.7\%, T ALL 0.9\%, HL 0.9\% and LCH $0.9 \%$. The study done in Eastern Morocco showed $\mathrm{HL}$ as the most frequent $\mathrm{HM}$ in this age group followed by respectively by NHL, MPN, AML, ALL and MM, CLL, MDS and WM. In our study NHL was the most frequent HM whereas other study showed HL as the commonest HM. ${ }^{7}$ In Bangladesh, AML has been commonly seen at this age group. ${ }^{8}$

In adults aged 41 to 60 years, MPN 12.6\% (CML 6.3\%, ET $2.7 \%$, PV1.8\%, PM1.8\%) was the most common HM followed respectively by $\mathrm{PCN}$ accounting $10.9 \%$ followed by AML $7.2 \%$, NHL $4.5 \%$, MDS 3.6\%, ALL $1.8 \%$, HL $0.9 \%$,CLL $1(0.9 \%)$ and LPL $1(0.9 \%)$. In this age group other study showed NHL was predominant HM followed by MPN, MM, HL, CLL, AML, MDS, ALL and WM. ${ }^{7}$ In our study MPN and PCN was the commonest HM in this age group whereas other study showed NHL as the commonest HM. CLL, MDS and PCN has been reported as common Hematological malignancies in this age group. ${ }^{8}$ hematological malignancies arising from mature immunocompetent cells (mostly B lineage) predominate in adults. ${ }^{15}$ A variation in hematological malignancies with age suggests that immune system rich in precursor cells in young people and predominance of germinal centre and memory B cells in older adults. ${ }^{15}$

In older patients of more than 61 years of age, PCN was the most common HM accounting $10.9 \%$ followed by NHL $3.6 \%$, AML $2.9 \%$, CLL $1.8 \%$, MPN 1.8\%(CML 0.9\%, ET $0.9 \%$ ) and MDS $1(0.9 \%)$. One of the study showed NHL as the most common HM followed by MM, MPN, CLL, 
HL, MDS, AML, ALL and WM. ${ }^{7}$ Our study shows PCN is the commonest HM whereas other study showed NHL as the commonest HM. It has been observed that in northen India; PCN (multiple myeloma) is more common in older age group with median age of 61 years. ${ }^{14}$

\section{Conclusion}

Hematological malignancies can occur at any age group and men are more commonly affected. Lymphoid neoplasm is the most frequent HM. NHL is the most common HM in young adults whereas PCN is the most common HM in elderly. Hematological malignancies arising from Precursors $\mathrm{T}$ and $\mathrm{B}$ cells are common in children and adolescents where as Lymphoid Neoplasm arising from germinal centre and memory B cells are common in adults.

\section{References}

1. John B(2004) Cousar Hematopoeitic-lymphoid Neoplasms: principles of diagnosis-In Greer JP, Rodgers GM,Foerster J, Paraskevas F, Lukens JN, Glader B, editors Wintrobe's Clinical Hematology. Vol2 11th ed. Philadelphia USA: Lipincott Williams \& Wilkins, 1914

2. Rowers CR Glover R lonial S, Brawely OW. Racial differences in the incidence and outcomes for patients with Hematological malignancies. Curr probl Cancer. 2007,31(3).182-201

3. Jaffe ES, Harris NL, Sten H, Vardiman JW. Pathology and genetics of tumors of hematopoietic and lymphoid tissues. World Health Organisation Classification of tumors. Lyon, France: larc Presse:2001.

4. Vadiman JW, Harris NL, Brunning RD. The World Health Organisation (WHO) classification of myeloid neoplasms. Blood. 2002;100(7)2292-302. https://doi. org/10.1182/blood-2002-04-1199;PMid:12239137.

5. Daniel A. Arber, Attilio Orazi, Robert Hasserijian. The Upadated WHO Classification of Hematological Malignancies. The 2016 revision to the WHO Health Organization classification of myeloid neoplasms and acute leukemia. Blodd.2016; vol 127,nov 20. 23912405

6. Steven H. Swerdlow, Elias Campo, Stefano A. The updated WHO Classification of Hematological Malignancies. The 2016 revision of world Health Organization classification of Lymphoid Neoplasms. Blood.2016; vol 127,nov 20. 2375-2390.

7. Mounia Elidrissi Errahhai, Manai Eldrissi, Redouane Boulouiz. Distribution and features of hematological malignancies in Eastern Morocco: a retrospective multicentre study over 5 years. BMC Cancer (2016)
16:159. https://doi.org/10.1186/s12885-016-2205-5 PMid:26915519 PMCid:PMC4768409

8. Hossain MS, Iqbal MS, Khan MA, Rabbani MG, KhatunH. Diagnosed Hematological malignancies in Bangladesh- a retrospective analysis of over 5000 cases from 10 specialised hospitals. BMC cancer.2014;14(1):438. https://doi.org/10.1186/14712407-14-438 PMid:24929433 PMCid:PMC4063230

9. Curado MP, Edwards B, Shin HR. Cancer Incidence in five continents,vol.9.International Agency for Research on Cancer;2007.

10. Sant M,Allemani C, Tereanu $C$ et al. Incidence of hematologic malignancies in Europe by morphologic subtype; results of HAEACARE project. Blood.2010;116(19):372-34. https://doi.org/10.1182/blood-2010-05-282632; PMid:20664057

11. Smith A, Howell D, Patmore R. Incidence haematological malignancy by subtype: a report from Haematological Malignancy Research Network. $\quad \mathrm{Br} \quad \mathrm{J}$ Cancer.2011;105(11):1684-92 https://doi.org/10.1038/bjc.2011.450; PMid:22045184 PMCid:PMC3242607

12. Thiam D, Diop S, Diop $\mathrm{T}$ et al.Epidemiology and therapy of malignant hemopathies in Snega. Hematol Cell Ther. 1996;38(2):187-91. https://doi.org/10.1007/ s00282-996-0187-5; PMid:8932001.

13. Troussard X,Duchenet Vet al. Haematological malignancies incidence in BasseNormandie, France, for 1997-2004. Rev Epideiol Sante Publique. 2009;57(3):151-8. https://doi.org/10.1016/j.respe.2009.02.204; PMid:19375876.

14. Anuradha Kusum, Gita Negi, DUshyant Singh Gaur, Sanjeev Kishore, Harsh Meena. Hematological Malignancies diagnosed by Bone marrow examination in a tertiary hospital at Uttarakhand, India. Indian J. Hematol.Blood Transfusions 24(1):7-11.

15. A Smith, D howel, $\mathrm{R}$ Patmore, A Jack and E Roman. Incidence of haematological malignancy by subtype: a report from the Haematological Malignancy Research Network. British journal of Cancer(2011).105,1684-1692.https://doi.org/10.1038/ bjc.2011.450. PMid:22045184 PMCid:PMC3242607

16. Shahtaj Khan, Awal Mir, Baber Rehman Khattak, Shujaat Gull. Pattern of adulthood hematological malignancies in Khyber Pakhtunkhwa. J Blood Disord Transfus 2018, vol 9. 